\title{
Least Squares Sparse Principal Component Analysis and Parallel Coordinates for Real-time Process Monitoring
}

\author{
Shriram Gajjar', Murat Kulahci ${ }^{2,3}$, Ahmet Palazoglu ${ }^{1, *}$ \\ ${ }^{1}$ Department of Chemical Engineering, University of California, Davis, CA 95616, USA \\ 2 Department of Applied Mathematics and Computer Science, Technical University of Denmark, Lyngby, Denmark \\ ${ }^{3}$ Department of Business Administration, Technology and Social Sciences, Luleå University of Technology, Luleå, Sweden
}

* Corresponding author. e-mail: anpalazoglu@ucdavis.edu

\section{$\underline{\text { Supplemental Information }}$}

Table S1: Confusion matrix obtained from test data for Random Forests model trained on 14 SPC and corresponding errors obtained with 70\% CPV LS SPCA dataset.

Figure S1. Fault \#6 signature in parallel coordinates for 70\% CPV LS SPCA.

Figure S2. Fault \#1 residual error signature in parallel coordinates for 70\% CPV LS SPCA.

Figure S3. Fault \#6 residual error signature in parallel coordinates for 70\% CPV LS SPCA. 
Table S1: Confusion matrix obtained from test data for Random Forests model trained on 14 SPC and corresponding errors obtained with $70 \%$ CPV LS SPCA dataset.

\begin{tabular}{|c|c|c|c|c|c|c|c|c|c|c|c|c|c|c|c|c|c|c|c|c|c|c|c|c|}
\hline & & \multicolumn{21}{|c|}{ Reference } & \multirow[b]{2}{*}{ Sum } & \multirow[b]{2}{*}{ Specificity } \\
\hline & & 1 & 2 & 3 & 4 & 5 & 6 & 7 & 8 & 9 & 10 & 11 & 12 & 13 & 14 & 15 & 16 & 17 & 18 & 19 & 20 & 21 & & \\
\hline \multirow[t]{21}{*}{ Predicted } & 1 & 184 & 0 & 0 & 0 & 0 & 0 & 0 & 0 & 0 & 0 & 0 & 0 & 0 & 0 & 0 & 0 & 0 & 0 & 0 & 0 & 0 & 184 & 1.00 \\
\hline & 2 & 0 & 183 & 0 & 0 & 0 & 0 & 0 & 0 & 0 & 0 & 0 & 0 & 0 & 0 & 0 & 0 & 0 & 0 & 0 & 0 & 0 & 183 & 1.00 \\
\hline & 3 & 0 & 0 & 92 & 0 & 0 & 0 & 0 & 1 & 20 & 5 & 3 & 0 & 0 & 0 & 17 & 3 & 2 & 3 & 6 & 3 & 0 & 155 & 0.98 \\
\hline & 4 & 0 & 0 & 0 & 170 & 0 & 0 & 0 & 0 & 0 & 0 & 7 & 0 & 0 & 0 & 0 & 0 & 0 & 0 & 0 & 0 & 0 & 177 & 1.00 \\
\hline & 5 & 0 & 0 & 0 & 0 & 156 & 0 & 0 & 0 & 0 & 0 & 0 & 0 & 0 & 0 & 0 & 0 & 0 & 2 & 0 & 0 & 0 & 158 & 1.00 \\
\hline & 6 & 0 & 0 & 0 & 0 & 0 & 163 & 0 & 0 & 0 & 0 & 0 & 0 & 0 & 0 & 0 & 0 & 0 & 0 & 0 & 0 & 0 & 163 & 1.00 \\
\hline & 7 & 0 & 0 & 0 & 0 & 0 & 0 & 169 & 0 & 0 & 0 & 0 & 0 & 0 & 0 & 0 & 0 & 0 & 0 & 0 & 0 & 0 & 169 & 1.00 \\
\hline & 8 & 0 & 0 & 0 & 0 & 0 & 0 & 0 & 171 & 0 & 0 & 0 & 1 & 1 & 0 & 0 & 0 & 0 & 0 & 0 & 0 & 0 & 173 & 1.00 \\
\hline & 9 & 1 & 1 & 34 & 0 & 0 & 0 & 0 & 2 & 109 & 9 & 3 & 1 & 8 & 0 & 19 & 9 & 0 & 13 & 5 & 12 & 0 & 226 & 0.97 \\
\hline & 10 & 0 & 0 & 5 & 0 & 1 & 0 & 0 & 1 & 4 & 127 & 1 & 5 & 0 & 0 & 2 & 8 & 0 & 0 & 0 & 2 & 0 & 156 & 0.99 \\
\hline & 11 & 0 & 0 & 0 & 3 & 0 & 0 & 1 & 0 & 0 & 1 & 152 & 0 & 0 & 0 & 2 & 1 & 1 & 0 & 6 & 0 & 0 & 167 & 1.00 \\
\hline & 12 & 0 & 0 & 0 & 0 & 0 & 0 & 0 & 2 & 0 & 1 & 0 & 177 & 4 & 0 & 0 & 0 & 0 & 1 & 0 & 0 & 0 & 185 & 1.00 \\
\hline & 13 & 0 & 0 & 0 & 0 & 0 & 0 & 0 & 0 & 0 & 0 & 0 & 3 & 154 & 0 & 0 & 0 & 0 & 0 & 0 & 0 & 0 & 157 & 1.00 \\
\hline & 14 & 0 & 0 & 0 & 0 & 0 & 0 & 0 & 0 & 0 & 0 & 4 & 0 & 0 & 171 & 0 & 0 & 0 & 0 & 0 & 0 & 0 & 175 & 1.00 \\
\hline & 15 & 0 & 0 & 35 & 0 & 0 & 0 & 0 & 0 & 26 & 11 & 15 & 0 & 3 & 0 & 123 & 9 & 3 & 5 & 11 & 17 & 0 & 258 & 0.96 \\
\hline & 16 & 0 & 0 & 4 & 0 & 0 & 0 & 0 & 0 & 1 & 9 & 2 & 0 & 0 & 0 & 5 & 117 & 0 & 0 & 0 & 7 & 0 & 145 & 0.99 \\
\hline & 17 & 0 & 0 & 1 & 0 & 0 & 0 & 0 & 0 & 1 & 1 & 0 & 0 & 0 & 11 & 0 & 1 & 175 & 0 & 0 & 0 & 0 & 190 & 1.00 \\
\hline & 18 & 0 & 0 & 0 & 0 & 0 & 0 & 0 & 0 & 0 & 0 & 0 & 0 & 0 & 0 & 0 & 0 & 0 & 144 & 0 & 0 & 0 & 144 & 1.00 \\
\hline & 19 & 0 & 0 & 9 & 0 & 0 & 0 & 0 & 0 & 3 & 4 & 2 & 0 & 0 & 0 & 10 & 4 & 0 & 0 & 141 & 1 & 2 & 176 & 0.99 \\
\hline & 20 & 1 & 0 & 5 & 0 & 0 & 0 & 0 & 0 & 6 & 1 & 0 & 0 & 0 & 0 & 2 & 2 & 0 & 1 & 3 & 133 & 2 & 156 & 0.99 \\
\hline & 21 & 0 & 0 & 2 & 0 & 0 & 0 & 0 & 0 & 0 & 0 & 0 & 0 & 0 & 0 & 0 & 2 & 0 & 0 & 0 & 1 & 173 & 178 & 1.00 \\
\hline & Sum & 186 & 184 & 187 & 173 & 157 & 163 & 170 & 177 & 170 & 169 & 189 & 187 & 170 & 182 & 180 & 156 & 181 & 169 & 172 & 176 & 177 & 3675 & \\
\hline
\end{tabular}


\begin{tabular}{|l|l|l|l|l|l|l|l|l|l|l|l|l|l|l|l|l|l|l|l|l|l|l|}
\hline Sensitivity & 0.99 & 0.99 & 0.49 & 0.98 & 0.99 & 1.00 & 0.99 & 0.97 & 0.64 & 0.75 & 0.80 & 0.95 & 0.91 & 0.94 & 0.68 & 0.75 & 0.97 & 0.85 & 0.82 & 0.76 & 0.98 & \\
\hline
\end{tabular} 


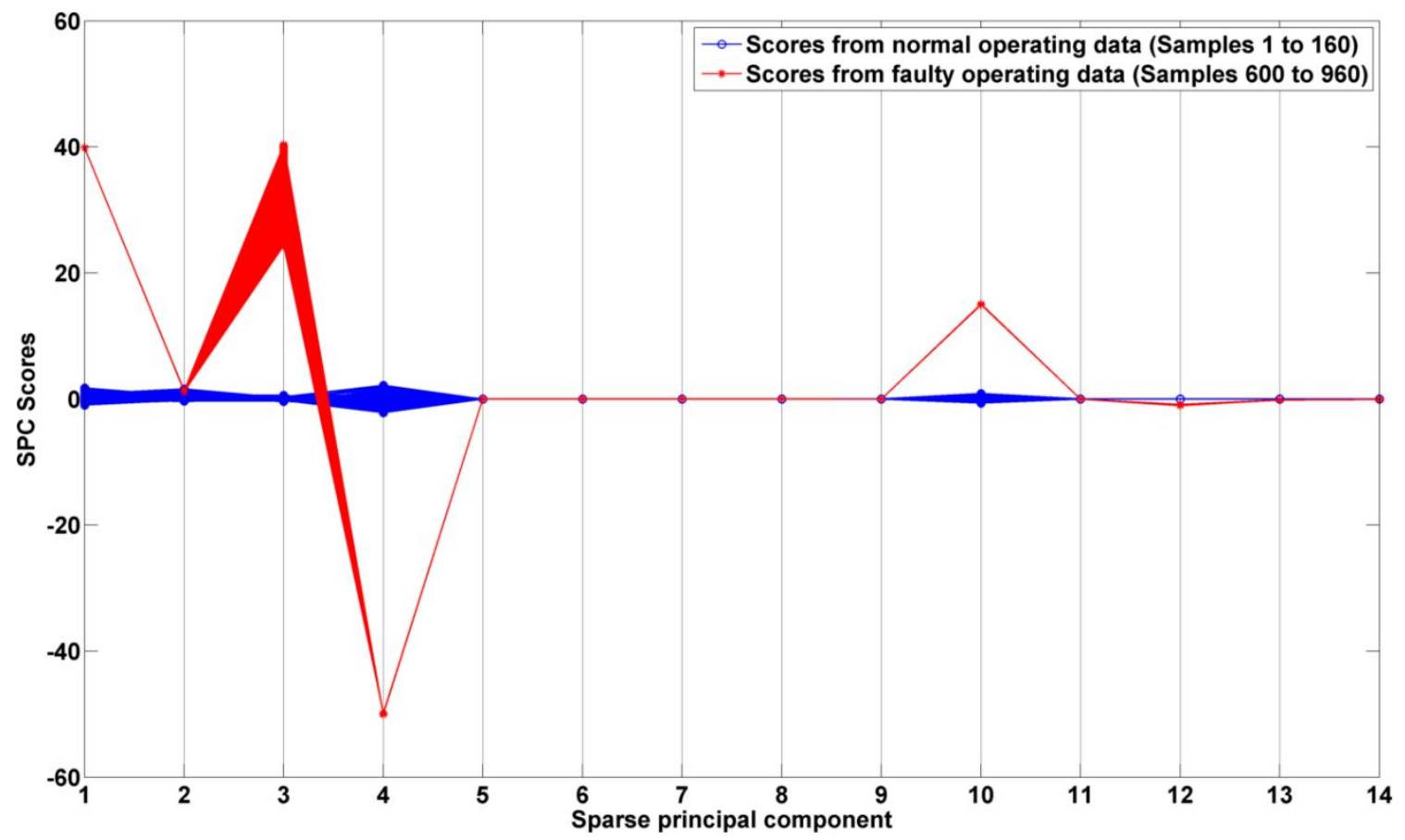

Figure S1. Fault \#6 signature in parallel coordinates for 70\% CPV LS SPCA. 


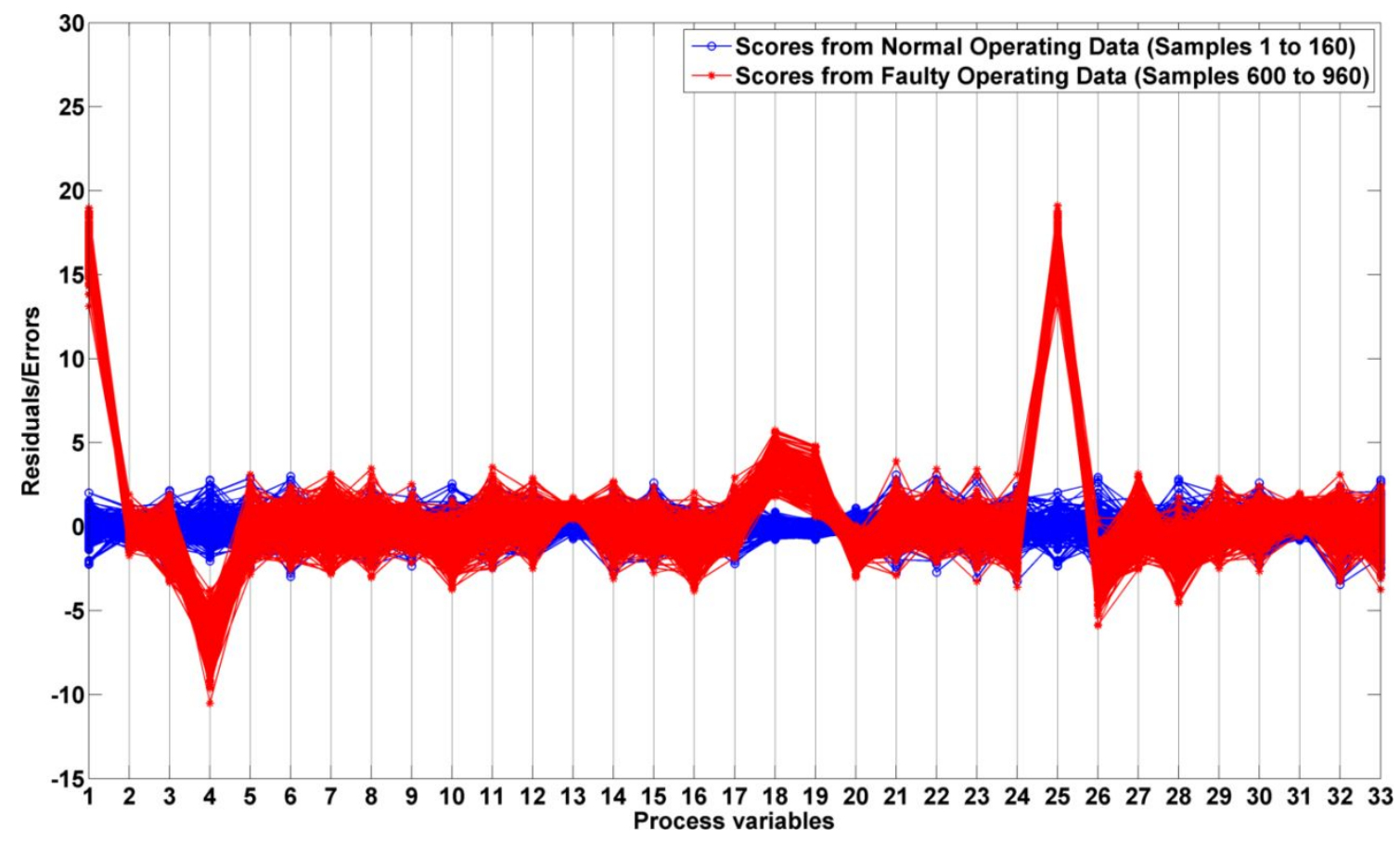

Figure S2. Fault \#1 residual error signature in parallel coordinates for 70\% CPV LS SPCA. 


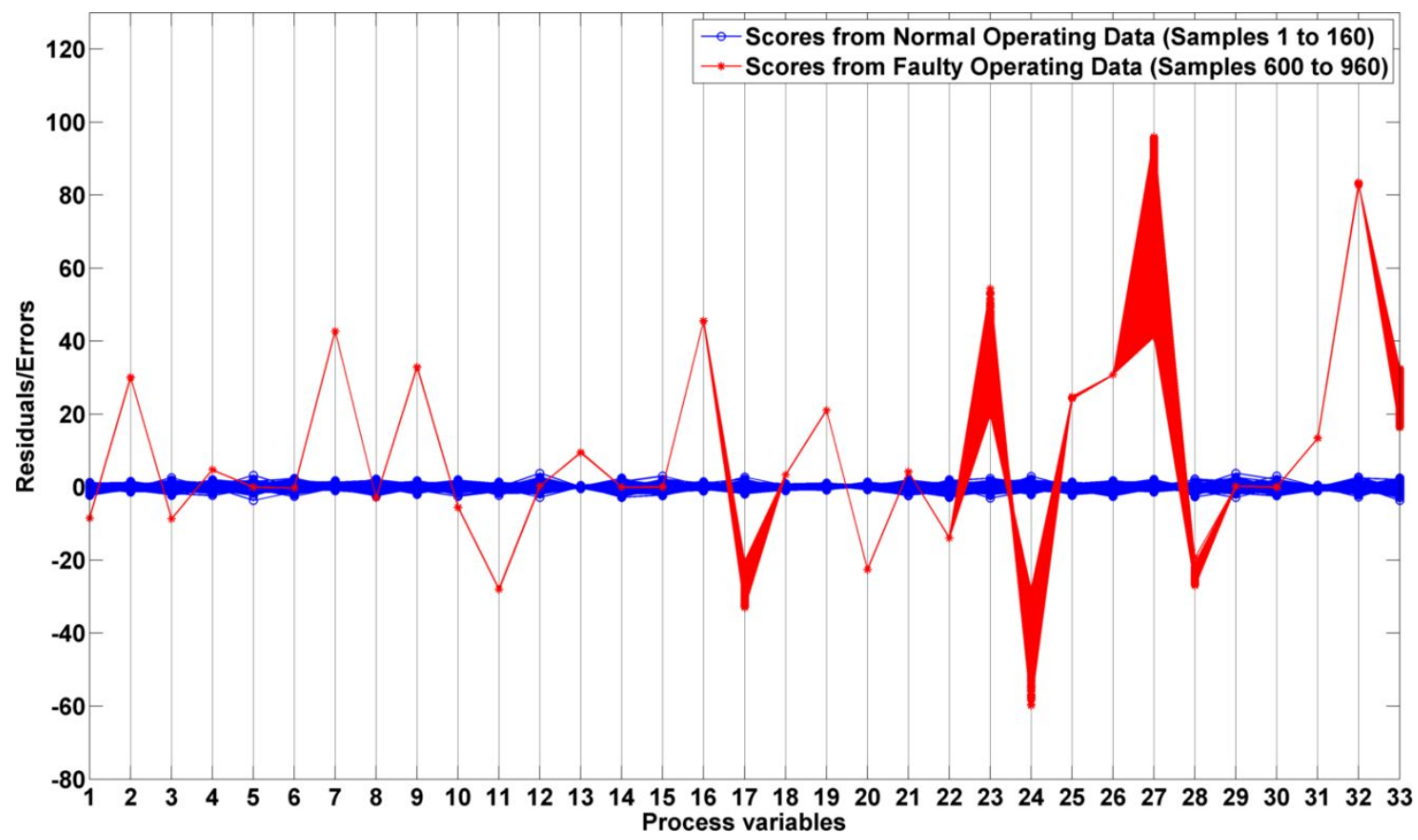

Figure S3. Fault \#6 residual error signature in parallel coordinates for $70 \%$ CPV LS SPCA. 